A resurrection study reveals limited evolution of thermal performance in response to recent climate change across the geographic range of the scarlet monkeyflower

6 Carolina

7 2. Department of Evolution and Ecology, University of California, Davis, California

$9 *$ Co-corresponding authors

10 Emails: rcwooliv@ncsu.edu, Seema_Sheth@ncsu.edu 
Abstract

12 Evolutionary rescue can prevent populations from declining under climate change, and should be

13 more likely at high-latitude, "leading" edges of species' ranges due to greater temperature

14 anomalies and gene flow from warm-adapted populations. Using a resurrection study with seeds

15 collected before and after a seven-year period of record warming, we tested for thermal

16 adaptation in the scarlet monkeyflower Mimulus cardinalis. We grew ancestors and descendants

17 from northern-edge, central, and southern-edge populations across eight temperatures. Despite

18 recent climate anomalies, populations showed limited evolution of thermal performance curves.

19 However, one southern population evolved a narrower thermal performance breadth by $1.25^{\circ} \mathrm{C}$,

20 which matches the direction and magnitude of the average decrease in seasonality experienced.

21 Consistent with the climate variability hypothesis, thermal performance breadth increased with

22 temperature seasonality across the species' geographic range. Inconsistent with performance

23 trade-offs between low and high temperatures across populations, we did not detect a positive

24 relationship between thermal optimum and mean temperature. These findings fail to support the

25 hypothesis that evolutionary response to climate change is greatest at the leading edge, and

26 suggest that the evolution of thermal performance is unlikely to rescue most populations from the

27 detrimental effects of rapidly changing climate.

28 Keywords: evolutionary rescue; geographic range; resurrection study; thermal adaptation;

29 thermal performance curve 
Introduction

31 Evolution can facilitate species persistence in the face of changing climate (Hoffmann and Sgrò

32 2011; Carlson et al. 2014), especially when extensive habitat fragmentation prevents migration

33 (Collingham and Huntley 2000) or plasticity is not sufficient to suit organisms to novel

34 environments (Visser 2008). Because climate change is causing mismatches between species'

35 geographic ranges and thermal niches, thermal adaptation is an important driver of population

36 responses to climate change (Geerts et al. 2015). Evolutionary rescue in the face of

37 environmental change occurs when adaptive evolution restores positive growth rates to

38 populations in decline, and it is most likely when the rate of environmental change is gradual and

39 the amount of standing genetic variation for ecologically important traits is high (Carlson et al.

40 2014). Yet, there is severe uncertainty regarding how extreme selection events associated with

41 changing climate (as opposed to gradual environmental change) will impact the extent to which

42 adaptive evolution can rescue populations in decline, and whether adaptive evolution varies

43 across species' geographic ranges.

44 Capacities for thermal adaptation may vary among populations across a species' range for

45 at least three reasons. First, populations may experience different magnitudes of climate

46 anomalies (departures of contemporary climate from historical averages), and thus different

47 selective pressures on thermal tolerance. For example, temperature increases associated with

48 climate change are often greater at higher latitudes relative to lower latitudes (IPCC 2013).

49 Second, populations may differ in the ability to evolve earlier phenology that would enable

50 avoidance of drought or extreme heat encountered during the growing season, consequently

51 relaxing selection for heat tolerance (Franks et al. 2007; Sheth and Angert 2016; Socolar et al.

52 2017; Dickman et al. 2019). In fact, empirical studies indicate that selection for early flowering 
53 can result in correlated reductions in stress tolerance (Franks 2011; Hamann et al. 2018). Third,

54 populations may differ in adaptive genetic variation due to connectivity with other populations.

55 High-latitude, leading-edge populations may have ample genetic variation to evolve as they

56 receive warm-adapted alleles from lower-latitude populations, but low-latitude, trailing-edge

57 populations may lack genetic variation due to a scarcity of populations adapted to warmer

58 temperatures (Davis and Shaw 2001; Hampe and Petit 2005; Hu and He 2006). Nonetheless,

59 recent work suggests that evolutionary rescue may not occur fast enough for populations to keep

60 up with the pace of climate change. The probability of evolutionary rescue may be especially low

61 if amelioration of climate extremes induces reversals in trait evolution (Hamann et al. 2018) or if

62 long generation times slow the rate of evolution (Hoffmann and Sgrò 2011). Ultimately,

63 understanding variation in thermal niche evolution among populations could improve models

64 that predict how species' distributions will shift with climate change, most of which currently

65 assume evolutionary stasis of species' climatic niches across space and time (Angert et al. 2011;

66 Hällfors et al. 2016; Peterson et al. 2019).

67 Temperature can shape species' distributions via its effects on fitness and other

68 performance metrics, yet we have only recently begun to understand the evolution of thermal

69 performance across space and time (Araújo et al. 2013; Diamond 2017). A thermal performance

70 curve (TPC) describes the performance of a genotype, population, or species across a

71 temperature gradient (Huey and Stevenson 1979; Angilletta 2009; Fig. 1A). A TPC peaks at an

72 intermediate temperature (thermal optimum) and is bounded by a temperature on either side

73 where performance falls to zero (upper and lower thermal limits). The span of temperatures

74 across which organisms achieve a designated percentage of the maximum performance is called 
75 the thermal performance breadth (hereafter referred to as breadth), and narrower breadth

76 suggests greater thermal specialization.

77 These TPC parameters, like many other traits such as phenology or resource acquisition,

78 can exhibit adaptive clines across spatial climatic gradients such as latitude (Lynch and Gabriel

79 1987; Angilletta 2009). Performance trade-offs between low and high temperatures, manifested

80 by shifts in the TPC along the temperature axis, yield the expectation that thermal optima

81 increase with environmental temperature (Angert et al. 2011). For example, thermal optima of

82 populations of Mimulus cardinalis in western North America increased with average July

83 temperatures and decreased with latitude, suggesting adaptive differentiation across the species'

84 range (Angert et al. 2011; Paul et al. 2011). If these patterns across space also apply across time,

85 climate change-induced increases in mean temperatures should result in the evolution of

86 increased thermal optima. The climate variability hypothesis posits that populations inhabiting

87 regions that are climatically stable should evolve narrower climatic tolerances relative to those

88 from climatically heterogeneous areas (Dobzhansky 1950; Janzen 1967; Stevens 1989). For

89 ectothermic animals, thermal breadth decreases towards the equator because organisms at lower

90 latitudes experience lower temperature variation (Sunday et al. 2010). This hypothesis has

91 primarily been tested in temperate-tropical comparisons, but also applies to situations where

92 climate variability changes across time, including recent climate change. That is, shifts in

93 seasonality related to climate change could result in the evolution of altered breadth. Although

94 there is some evidence of rapid evolution of thermal performance in response to climate change

95 in vertebrates and invertebrates (Kingsolver et al. 2013; Higgins et al. 2014; Geerts et al. 2015),

96 little is known about the evolution of TPCs in response to climate change in plants. 
To explore how thermal performance has evolved in response to recent climate change

98 and how the direction and magnitude of shifts vary across populations, we implemented a

99 resurrection experiment (Franks et al. 2008) with populations across the broad geographic range

100 of the scarlet monkeyflower, Mimulus cardinalis (Lowry et al. 2019). For each of two northern-

101 edge, two central, and two southern-edge populations that collectively span broad climatic

102 gradients in western North America (Fig. 2), we grew ancestors from 2010 alongside

103 descendants from 2017 in growth chambers. Ancestors and descendants were respectively

104 derived from seed collected before and after a seven-year period of record-setting drought and

105 heat in western North America. Specifically, northern and central populations experienced the

106 most extreme increases in temperature relative to historical conditions (Fig. 2B; Table 1).

107 Further, temperature seasonality and annual precipitation decreased substantially in southern

108 populations in recent years (Fig. 2C; Tables 1, S1). Recent population declines, coupled with low

109 probabilities of survival and high probabilities of reproduction at the southern range edge,

110 suggest that drought and warming could select for an "annualized" life history in this perennial

111 species (Sheth and Angert 2018). Thus, decreased generation times could enhance the potential

112 for evolutionary responses in some populations. We performed growth chamber experiments in

113 eight temperature regimes to build TPCs for ancestors and descendants within each population.

114 Because we held all aspects of the environment other than temperature constant, and produced

115 seed families for both ancestors and descendants in a common environment, we can confidently

116 attribute differences in TPCs between ancestors and descendants to genetic changes, rather than

117 plastic developmental responses, seed storage/age effects, or maternal effects (Franks et al. 2018,

118 2019). We tested two hypotheses about evolutionary responses of TPCs to climate change. First,

119 under directional warming, particularly in northern and central populations, increased thermal 
120 optima should evolve (Table 1; Fig. 1B). Second, under lower temperature seasonality,

121 particularly in southern populations, decreased breadth should evolve (Table 1; Fig. 1C).

122 Differences in evolutionary change in these TPC parameters among populations would suggest

123 that thermal adaptation is dependent upon variation in climate anomalies, evolution of avoidance

124 traits, and/or genetic variation in thermal performance. We also explored whether thermal optima

125 and breadths are associated with geographic temperature gradients, allowing us to test for

126 evolutionary divergence in thermal performance parameters across space.

Methods

130 Mimulus cardinalis is a perennial herb that occurs along seeps and streams from central Oregon,

131 USA to northern Baja, Mexico (Fig. 2A). It has been the subject of numerous investigations of

132 local adaptation, geographic range limits (Angert and Schemske 2005; Angert 2006; Paul et al.

133 2011; Muir and Angert 2017; Angert et al. 2018; Bayly and Angert 2019), and physiological,

134 evolutionary, and demographic responses to climate change (Angert et al. 2011; Sheth and

135 Angert 2016, 2018). Previous work indicates that populations in our study have been in decline,

136 but differences in generation time and gene flow across the range may affect the potential for

137 evolutionary rescue. Specifically, a demography study showed that during a five-year period of

138 severe drought and warming (2010-2014), growth rates of 32 M. cardinalis populations

139 decreased from the leading to trailing edges of the geographic range (Sheth and Angert 2018).

140 Three of these populations are included in our study (N1, C2, and S1; Table 1), and each of these

141 showed a significant population decline during the study period (Sheth and Angert 2018).

142 Further, the demography study showed that the probability of survival from one year to the next 
143 was highest in central populations and declined towards northern and southern range edges. A

144 majority of adults marked in 2010 in N1 and S1 populations did not survive to 2011 (Sheth and

145 Angert 2018), and data collected beyond 2014 suggest that a few C2 plants could survive at least

146 6-7 years (Angert and Sheth unpubl. data). Thus, populations in the range center likely have

147 longer generation times and lower potential for rapid evolution than those at range edges. A

148 genetic study of the northern half of the range of $M$. cardinalis showed that northern populations

149 have recently received a net influx of migrants from hotter environments (Paul et al. 2011),

150 which could enhance genetic variation in thermal performance and facilitate adaptation to a

151 warming climate.

152 We collected seeds from 80-100 individuals in each of the six study populations in 2010

153 (ancestors) and 2017 (descendants). Ancestors were collected as described in Sheth and Angert

154 (2016), and descendants were re-collected using the same protocol. Although there is a

155 possibility that a seedbank could have introduced individuals into the descendent populations

156 whose parents were not exposed to the period of anomalous climate during the study period (i.e.,

157 pre-2010), previous observations have pointed towards limited seed dormancy in M. cardinalis.

158 In particular, a study of mid-latitude populations (2002-2003) found that only a small fraction of

159 seeds can remain viable in the seed bank for at least one year (Angert 2006), but a recent study of

1607 populations spanning the latitudinal range (2011-2014) demonstrated that no germination

161 occurred after the first year in the seed bank (Sheth and Angert 2018). To minimize maternal and

162 storage/age effects, we grew seeds in the greenhouse for a 'refresher' generation and performed

163 controlled crosses to produce 18 seed families within each population/cohort combination

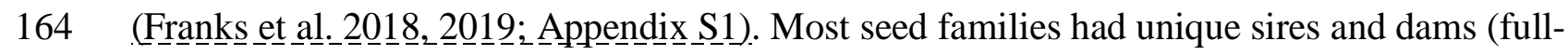

165 sibs), with the exception of some crosses that shared the same sire (half-sibs) in four 
166 population/cohort combinations with low parental sample sizes (N1 2010, N2 2010, C2 2010,

167 and C2 2017; Table S2).

\section{Resurrection experiment}

169 To determine whether M. cardinalis TPCs have evolved in response to recent climate change

170 across the geographic range, we implemented a resurrection experiment in growth chambers

171 using ancestral and descendent seed families of the six populations from the refresher generation

172 (Appendix S1). In summary, we grew seedlings in one of eight 16h day/8h night temperature

173 regimes $\left(10 /-5,15 / 0,20 / 5,25 / 10,30 / 15,35 / 20,40 / 25\right.$, or $\left.45 / 30{ }^{\circ} \mathrm{C}\right)$ for one week. These

174 temperature regimes encompass temperatures experienced by each of the six populations (Fig.

175 S1) and capture full TPCs for M. cardinalis and close relatives (Paul et al. 2011; Sheth and

176 Angert 2014). Previous work in Mimulus has documented substantial variation in growth across

177 temperatures during the short time frame of one week (Paul et al. 2011; Sheth and Angert 2014).

178 In each growth chamber run, we included seedlings from each of the 18 seed families within

179 each of the 12 population/cohort combinations. Temperature regimes were replicated twice to

180 reduce chamber effects. Each seed family was replicated four times in each temperature regime,

181 with two replicates in each chamber run $(6,912$ plants total).

182 Prior to chamber runs, we planted seeds into 72-cell plug trays. We planted into sets of

183 six trays, which together eventually went into each growth chamber run and contained the two

184 replicate plants for all 216 seed families planted in a randomized design. Two to ten seeds were

185 planted for each replicate. Seeds were germinated under a benign day/night temperature regime

$186\left(20 / 15^{\circ} \mathrm{C}\right)$ and a 16-hour photoperiod (6:00-22:00). Three to four weeks after planting, when

187 most seedlings had germinated but were small enough that roots were not yet entangled, we

188 thinned seedlings to one central-most seedling in each cell. Two weeks after thinning, when most 
seedlings had at least two true leaves, we put each tray set (i.e., six trays containing two

190 replicates of all seed families) into one of four reach-in growth chambers (Percival LT-105X,

191 Percival Scientific, Inc., Perry, Iowa, USA) that was set to one of the eight day/night temperature

192 regimes (Appendix S1).

193 We measured the performance of all seedlings based on the relative change in leaf

194 number over the course of growth chamber runs. We recorded the number of true leaves $>1 \mathrm{~mm}$

195 in length on each individual immediately prior to, and one week after, being placed in the growth

196 chamber (lea $f_{\text {in }}$ and lea $f_{\text {out }}$, respectively). We then calculated relative growth rate (RGR) as:

$$
\frac{\left(\text { lea } f_{\text {out }}-\text { lea } f_{\text {in }}\right)}{\left(\text { lea } f_{\text {in }} \times \text { number of days }\right)}
$$

197 This metric does not incorporate total fitness in terms of reproductive output, and alternative

198 performance metrics could yield different results. However, size is positively related to fruit

199 number in natural populations of M. cardinalis (Sheth and Angert 2018). Because rapid growth

200 at early life stages during which plants are smaller and more vulnerable should increase the

201 chances of juvenile survival and thus the probability that a plant will reproduce, relative growth

202 rate is likely correlated with total fitness. Nonetheless, we emphasize that relative growth rate is

203 a metric of plant performance that is a component of fitness, rather than a metric of total fitness.

204 We excluded 866 plants that died, did not germinate, or did not have true leaves by the start of

205 chamber runs, and 13 that were accidentally damaged before final leaf number was

206 measured. Thus, at the end of the experiment, we measured RGR for 6,033 plants (of the 6,912

207 initially planted). For 718 individuals that died during chamber runs $\left(59 \%\right.$ of plants at $10 /-5^{\circ} \mathrm{C}$,

$20825 \%$ of plants at $45 / 30{ }^{\circ} \mathrm{C}$, and $<1 \%$ of plants at each of the other temperature regimes), we set

209 RGR equal to zero. No individual had fewer leaves coming out of the chamber than going in, so

210 all RGR values were greater than or equal to zero. 
212 We used RGR data to build TPCs for ancestors and descendants within each of the six $M$.

213 cardinalis populations. To determine how thermal performance has evolved in response to recent

214 climate change across the species' range, we compared thermal optima and breadths of ancestors

215 vs. descendants within each population using probabilistic comparisons (i.e., the proportion of

216 times that the parameter for a descendent group was greater than its respective ancestral group).

217 We used a hierarchical Bayesian model (R package performr v0.2;

218 https://github.com/silastittes/performr; Tittes et al. 2019) to fit TPCs to our data. This method

219 allowed us to simultaneously estimate responses (RGR) of our 12 biological groups (6

220 populations $\mathrm{x} 2$ cohorts) across an environmental gradient (daytime temperature) using a

221 derivation of Kumaraswamy's probability density function. There are two limitations to the

222 model in its current form. First, although there are more complex ways to model RGR as change

223 in leaf number (Rees et al. 2010), the model is unable to handle complex response variables.

224 Second, the model does not allow for random effects. Thus, prior to model implementation, we

225 averaged RGR among replicates of each family in each temperature regime to avoid

226 pseudoreplication within families and to minimize growth chamber effects $(\mathrm{N}=1,717$; Table S2).

227 We scaled RGR by the overall mean and centered daytime temperature around zero to improve

228 model performance. We used the default model settings, except we increased iterations per chain

229 to 10,000 and max_treedepth to 12 . These settings increased convergence and reliability of

230 posterior sampling according to $\hat{R}$ statistics of 1 and large numbers of effective samples (at least

231 10,000; Table S3; Gelman et al._2014). While we did not compare our model to alternatives, we

232 quantified the adequacy of the model's fit to the data using a Bayesian p-value (Gelman et al.

233 2014). An adequate model should have a Bayesian p-value near 0.5, indicating a lack of 
234 discrepancy between the data generated by the model and the empirical data. To compute the

235 Bayesian p-value, we compared 1) the sum of squares between the model's expected value and

236 the RGR values generated from each model's posterior draws, and 2) the sum of squares when

237 comparing posterior expectations to the empirical data. The Bayesian p-value was calculated as

238 the proportion of posterior draws where the former sum of squares was greater than the latter.

239 When simulating RGR values, all negative predictions were set to zero. The Bayesian p-value of

240 our model was 0.19 (Fig. S2), indicating that the model adequately described the data generating

241 process. Bayesian p-values for each population and cohort combination were also generally close

242 to 0.5 , though there were a few exceptions (Fig. S2). Lack of strong correlations in posterior

243 draws between the core parameters of this model indicate that variance inflation does not

244 influence posterior uncertainty (Fig. S3).

245 We derived thermal performance parameters of interest (thermal optimum and breadth)

246 from each posterior draw of the TPC model and placed the parameters back in their original scale

247 to aid interpretation. We calculated thermal optimum as the temperature at which RGR is

248 maximized (Tittes et al. 2019), and breadth as the range of temperatures across which plants

249 achieved at least $50 \%\left(\mathrm{~B}_{50}\right)$ and $80 \%\left(\mathrm{~B}_{80}\right)$ of maximum performance (Huey and Stevenson

250 1979). We calculated breadth by finding the approximate lower and upper temperature values

251 that corresponded to $50 \%$ or $80 \%$ of the maximum height of the curve. Lacking an exact solution

252 for the critical values, we selected them from a grid of 100 equally spaced points along the

253 temperature axis, choosing the two points that had the minimum distance to the desired

254 percentage of curve height. Results were qualitatively similar for $\mathrm{B}_{50}$ and $\mathrm{B}_{80}$ (Fig. S4B-C), so

255 we report results for only $\mathrm{B}_{50}$ along with differences in results in cases where they influence

256 interpretation. We performed pairwise comparisons of thermal optimum and breadth among all 
25712 population-by-cohort groups, focusing on comparisons between cohorts within populations.

258 Specifically, we calculated the average and 95\% credible interval of the difference in the

259 predicted parameter estimate of ancestors vs. descendants of each population. A 95\% credible

260 interval that did not overlap zero would indicate a highly plausible difference between

261 descendants and ancestors (i.e., we had the statistical power to detect evolutionary change). A

$26295 \%$ credible interval that did overlap zero would indicate that evolutionary change was not

263 highly plausible.

264 To test for evolutionary divergence in thermal optima and breadth across the geographic

265 temperature gradient, we implemented two linear models using the functions $I m$ and anova from

266 the stats package in R. We estimated means of thermal optima and breadths for each population

267 and cohort combination from posterior draws of the TPC model. Historical maximum July

268 temperature and historical temperature seasonality (maximum temperature of the warmest month

269 minus minimum temperature of the coolest month) were estimated for each population and

270 cohort combination as means from the years 1951-2000 (Table S1). The first model predicted

271 thermal optimum as a function of maximum July temperature, cohort, and their interaction. A

272 positive relationship between thermal optimum and maximum July temperature would confirm

273 that there are performance trade-offs between low and high temperatures. The second model

274 predicted breadth as a function of seasonality, cohort, and their interaction. A positive

275 relationship between breadth and seasonality would support the climate variability hypothesis.

276 For both models, we removed interactive and/or main effects of cohort when they were not

277 significant at $\alpha<0.05$. We used R v3.6.1 for all analyses (R Core Team 2019). 
Results

281 Overall, there was no support for the hypothesis that populations have evolved higher thermal

282 optima. The thermal optimum increased by averages of about $0.5^{\circ} \mathrm{C}$ from ancestors to

283 descendants in three populations — one population from each of the northern edge (N1), central

284 (C1), and southern edge (S1) regions of the geographic range (Figs. 3, 4A, S4A; Table 1).

285 Thermal optimum decreased in each of the three other populations (N2, C2, and S2) by averages

286 of less than $0.5^{\circ} \mathrm{C}$ (Figs. 3, 4A, S4A; Table 1). However, because the credible intervals for all

287 shifts in thermal optimum (both positive and negative) included 0 , we inferred that evolutionary

288 change was not highly plausible. Means and 95\% credible intervals for thermal optima and other

289 TPC parameters for all population/cohort combinations are reported in Table S4.

\section{Evolution of thermal performance breadth}

291 We found mixed support for the second hypothesis that populations have evolved narrower

292 breadths. On average, breadth increased in two populations (N1 and C2) and decreased in the

293 other four populations (Figs. 3, 4B, S4B; Table 1). Among these, the only highly plausible

294 evolutionary change detected was for S1, whose descendants had an average breadth that was

$2951.25^{\circ} \mathrm{C}$ narrower than ancestors (Figs. 3, 4B, S4B; Table 1). When comparing breadth at the

$29680 \%$ threshold $\left(\mathrm{B}_{80}\right)$, the direction of evolutionary change from ancestors to descendants was the

297 same as $B_{50}$ for each population, but that of S1 was no longer highly plausible (descendants had

298 an average $\mathrm{B}_{80}$ that was $0.87^{\circ} \mathrm{C}$ narrower than ancestors; Fig. S4C).

299 Evolutionary divergence in thermal optimum and breadth across temperature gradients

300 Overall, thermal optimum did not significantly vary with maximum July temperature

$301\left(F_{1,8}=0.744, p=0.414\right)$ or between cohorts $\left(F_{1,8}=0.236, p=0.640\right)$. The relationship between 
302 thermal optimum and maximum July temperature did not differ between cohorts $\left(F_{1,8}=0.0001\right.$,

$303 p=0.991$ ). After removing cohort as a main effect in the model, maximum July temperature

304 explained no variation in thermal optimum ( $b=0.063, R_{a d j}^{2}=-0.009, p=0.364$; Fig. $\left.4 \mathrm{~A}\right)$.

305 Breadth showed no significant differences between ancestors and descendants

$306\left(F_{1,8}=0.384, p=0.552\right)$, nor did breadth vary differently with seasonality between cohorts

$307\left(F_{1,8}=1.877, p=0.208\right)$. However, breadth increased with seasonality overall $\left(F_{1,8}=6.648\right.$,

$308 p=0.033)$. After removing cohort as a main effect in the model, seasonality explained $39 \%$ of the

309 variation in breadth $\left(b=0.253, R_{a d j}^{2}=0.386, p=0.018\right.$; Fig. 4B).

\section{Discussion}

312 We combined a resurrection study with a hierarchical Bayesian model to test key hypotheses

313 about how thermal performance has evolved in response to recent climate change across a plant

314 species' range. Since the introduction of resurrection studies over a decade ago (Franks et al.

315 2008), this is the first to test whether plant TPCs can rapidly evolve in response to contemporary

316 climate change. In only seven years encompassing anomalous temperatures and record drought,

317 we detected rapid evolution of the TPC in a southern, trailing-edge population of a perennial

318 herb. However, we show that rapid evolution is the exception rather than the norm across

319 multiple populations. Overall, there was no support for the hypothesis that the populations have

320 evolved higher thermal optima, and little support for the hypothesis that the populations have

321 evolved narrower breadths. One southern-edge population evolved a narrower breadth, indicating

322 increased thermal specialization. There was no apparent evolutionary change in the breadth of

323 northern-edge and central populations and the other southern-edge population. Below, we

324 interpret these findings in light of genetic variation, natural selection, and the evolution of 
325 avoidance traits across the species' geographic range, and we discuss their implications for

326 evolutionary rescue.

328 Genetic variation in thermal performance within populations and selective pressures from recent

329 trends in climate may explain the observed evolutionary shifts in TPCs. Directional warming,

330 estimated as the average anomaly in maximum July temperature during the study period, was

331 greatest in the central and northern populations (Table 1; Fig. 2B). Further, anomalies in

332 maximum July temperature steadily increased from 2010 to 2017 across populations (Fig. S5A,

333 Appendix S2), reducing the likelihood that amelioration in climate would have induced reversals

334 in trait shifts. Thus, upward evolutionary shifts in thermal optima (Fig. 1B) should have been

335 greatest in the central and northern populations if they are successfully adapting to climate

336 change. However, we did not detect significant increases in thermal optima in these populations

337 (Table 1; Figs. 3, S4A). One interpretation of this result is that evolutionary rescue is not

338 occurring rapidly enough for these populations to keep up with the pace of rapid climate change

339 (Hamann et al. 2018).

340 There are multiple potential explanations for the lack of evolutionary responses of

341 thermal optimum. First, climate-driven selection on thermal performance may not have been

342 strong enough to cause significant directional change in thermal optima. Average anomalies in

343 maximum July temperature were negative in southern populations (Table 1), so selection for

344 higher thermal optima could have been weak. In central and northern populations, average

345 anomalies in maximum July temperature were positive but less than $1{ }^{\circ} \mathrm{C}$ across populations.

346 Ancestors within all four of these populations had a thermal optimum that is at least $1{ }^{\circ} \mathrm{C}$ greater

347 than their respective historical maximum July temperatures (Fig. 4A; Tables S1, S4). Thus, 
ancestors were already equipped to tolerate the increased temperatures experienced throughout

349 the study period. Three populations showed shifts in thermal optima that were opposite to their

350 respective anomalies in maximum July temperature (N2, C2, and S1; Table 1). However, it is

351 worth noting that $\mathrm{C} 1$ - whose ancestors had the lowest thermal optimum of all populations

$352\left(31.71{ }^{\circ} \mathrm{C}\right)$ and experienced the greatest average increase in maximum July temperature $(+0.66$

$\left.353{ }^{\circ} \mathrm{C}\right)$ - showed a similar average increase in thermal optimum $\left(+0.63{ }^{\circ} \mathrm{C}\right.$; Table 1$)$, though the

354 credible intervals for this shift in thermal optimum overlap zero. Two other populations showed

355 an increase in thermal optimum that matched the magnitude of increase in maximum July

356 temperature (N1 and S2). Interestingly, although S2 did not experience maximum July

357 temperatures during the study period that were greater than historical averages, both cohorts

358 within $\mathrm{S} 2$ have a thermal optimum that is almost $1{ }^{\circ} \mathrm{C}$ less than its historical maximum July

359 temperature average (Fig. 4A; Tables S1, S4), and thus there may still be future selection for

360 higher thermal optimum. Given more time under elevated temperatures, N1, C1, and S2 may

361 have the greatest potential to track further increases in mean temperature through a shift in

362 thermal optimum.

364 constrain the evolution of thermal optima across the species' range. Northern populations have

365 recently received an influx of migrants from central populations that occur in hotter temperatures

366 (Paul et al. 2011). However, contemporary populations owe less than $1 \%$ of their genotypes to

367 recent migrants (i.e., within the last two generations; Paul_et_al_ 2011). Further, the limited gene

368 flow from central to northern populations that may have occurred over our study period may not

369 have introduced genotypes with higher thermal optima. This is because central populations,

370 though they occur in hotter environments (Fig. 2A), did not have higher thermal optima than 
371 northern populations (Figs. 4A, S4A). However, on average, the southern populations in our

372 study had higher thermal optima than central populations (Figs. 4A, S4A). One potential

373 explanation for this pattern is limited gene flow between southern and central populations, which

374 has preliminarily been shown by a range-wide population genetics dataset of M. cardinalis (J. R.

375 Paul, T. Parchman, A. Buerkle, and A. L. Angert, unpublished manuscript). Limited gene flow

376 between southern and central populations, paired with our finding that central populations have

377 not evolved higher thermal optima (Table 1), suggests that gene flow from southern populations

378 has not enhanced adaptation to warmer temperatures in central populations. Further, the southern

379 populations in our study may not have evolved higher thermal optima because their location

380 within the geographic range limits the opportunity to receive alleles from warmer-adapted

381 populations. A third reason for lack of evolution of thermal optimum is that ancestors lack

382 genetic variation in thermal optimum, a possibility that we are currently assessing. Overall, these

383 results indicate that evolutionary rescue has not yet occurred in $M$. cardinalis populations that

384 have declined during years of severe warming and drought. Evolution of thermal optima may not

385 have played an important role in buffering against $M$. cardinalis population declines in response

386 to recent climate change, but further work is needed to assess whether populations are able to

387 evolve in the long term.

According to the climate variability hypothesis, populations that experienced the lowest

389 temperature variation relative to historical averages should exhibit the greatest decreases in

390 breadth (Dobzhansky 1950; Janzen 1967; Stevens 1989). When climate is stable within the

391 lifetime of organisms, genotypes with high performance within the narrow climatic gradient are

392 favored (Etterson 2004). In support of the climate variability hypothesis, seasonality, which

393 represents the span of temperatures experienced during the year, was on average dramatically 
394 lower than historical conditions in the S1 population (Table 1; Fig. 2C), and this population

395 showed plausible evolution towards thermal specialization (i.e., breadth became narrower in

396 descendants relative to ancestors; Figs. 3, S4B). Two factors aside from decreased seasonality

397 could have contributed to the evolution of thermal specialization in S1. First, southern

398 populations of $M$. cardinalis have become increasingly annualized (i.e., high probabilities of

399 flowering and low probabilities of survival from one year to the next; Sheth and Angert 2018).

400 Annualization could enhance the rate of evolution due to shorter generation times. Second, S1

401 has experienced recent drought, receiving on average $111 \mathrm{~mm}$ less precipitation per year

402 compared to the historical average (the strongest drought across all six populations; Table S1).

403 Heat compounded by drought may have truncated the growing season and reduced the range of

404 temperatures the population encounters, thus increasing selection for thermal specialization.

405 Lower annual precipitation could also explain trends towards decreasing breadth in two other

406 populations, S2 and C1. Average anomalies in seasonality have been small but positive in the

407 northern populations (Table 1; Fig. 2C). Inconsistent with the climate variability hypothesis,

408 these northern populations did not evolve broader TPCs (Table 1; Figs. 3, S4B). However,

409 seasonality of each study year was not always greater compared to historical conditions and did

410 not follow a unidirectional trend across the study period (Figs. 2C, S5B), so it is possible that

411 selection for greater breadth was neither strong nor consistent across years. Further, populations

412 whose breadth did not evolve may have lacked standing genetic variation in breadth. Overall,

413 these results suggest that in $M$. cardinalis, breadth may be a more evolutionarily labile trait than

414 thermal optimum, but lack of breadth evolution in response to recent climate change in a

415 majority of populations indicates that evolutionary rescue has not occurred in populations that

416 are declining as temperatures have become more variable. 
Although there was limited evolution of thermal optimum and breadth from ancestors to

418 descendants, we found mixed support for our hypotheses across space. Despite limited gene flow

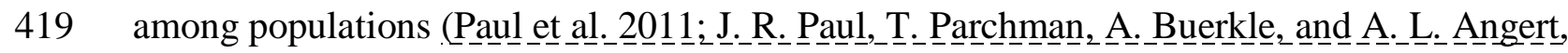

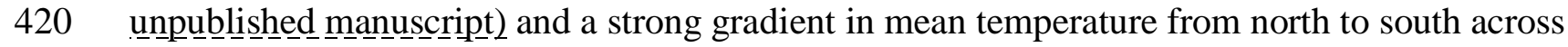

421 the geographic range (Fig. 2B), we did not detect a strong pattern of adaptive divergence in

422 thermal optima across the geographic mean temperature gradient (Fig. 4A). Interestingly, this

423 pattern contradicts previous work showing that thermal optima of $M$. cardinalis populations

424 increased from the northern range edge towards the range center (Angert et al. 2011; Paul et al.

425 2011). Further, though our results provide mixed support for the climate variability hypothesis

426 over time (no increase in breadth in two northern populations that experienced increased

427 seasonality, but decreased breadth in only one of four central and southern populations that

428 experienced decreased seasonality), our data strongly support the climate variability hypothesis

429 across the geographic temperature gradient. Contrary to the expected pattern of increased

430 seasonality from low to high latitudes, central and southern populations experience greater

431 seasonality and have broader TPCs than northern populations (Figs. 4B, S4B). Overall, breadth

432 increased with historical seasonality independent of cohort (Fig. 4B), indicating that there is

433 adaptive divergence in breadth across temperature variation, and this genetic cline is maintained

434 with contemporary evolution.

436 We have shown that only one out of six M. cardinalis populations has responded to recent

437 climate change through evolution of a narrower TPC (Table 1; Figs. 3, S4A-B). The populations

438 exhibiting no TPC evolution could instead persist under climate change through the evolution of

439 avoidance traits (Franks et al. 2007; Sheth and Angert 2016; Socolar et al. 2017; Dickman et al. 
440 2019). At the same time, these avoidance traits, including earlier flowering, may come with the

441 cost of lower tolerance to environmental stress. For example, when populations of Brassica rapa

442 evolved earlier flowering after a multi-year drought, they concurrently evolved decreased water-

443 use efficiency, and thus lower ability to tolerate drought conditions (Franks 2011; Hamann et al.

444 2018). Using the ancestral populations from our study, Sheth and Angert (2016) quantified the

445 response to artificial selection for early and late flowering as a proxy for each population's

446 adaptive potential. They found that the two southern populations rapidly responded to selection

447 on flowering time, with early- and late-flowering selection lines diverging by $\sim 15$ days. Thus,

448 the ancestors of the southern populations had ample genetic variation to evolve earlier flowering

449 times, potentially allowing them to avoid the extreme drought and increased temperatures that

450 they have recently experienced (Table S1). Additionally, early-flowering selection lines, though

451 they did not have decreased water-use efficiency, had higher specific leaf area and leaf nitrogen

452 content, representing a partial shift toward a fast, resource-acquisitive life history (Sheth and

453 Angert 2016). Thus, if southern populations have evolved earlier flowering in situ since 2010

454 (which we are currently assessing), they may have also evolved acquisitive life histories at the

455 expense of more resource-conservative functional traits that would promote thermal tolerance. In

456 line with this prediction, we found that the both southern populations tended to evolve narrower

457 TPCs (though not significant in S2; Table 1; Figs. 3, S4B), meaning that the descendants did not

458 tolerate extreme temperatures as well as their ancestors. Populations could have exhibited

459 evolutionary shifts in other TPC parameters, including lower and upper thermal limits, critical

460 breadth (the difference between upper and lower limit), performance maximum, and area under

461 the performance curve. For example, if S1 evolved a more competitive growth strategy, we

462 might predict that it has evolved a higher performance maximum. Though descendants of the 
463 southern populations on average had a higher performance maximum than their respective

464 ancestors, these shifts were not highly plausible (Figs. 3, S4G; Table S4). In fact, with the

465 exception of decreased breadth in S1, we did not detect highly plausible shifts in any TPC

466 parameter for any of our study populations (Fig. S4). Thus, our results provide mixed evidence

467 for a clear evolutionary trade-off between the ability to avoid novel environments and

468 environmental tolerance, at least over the seven-year study period.

469 Though southern populations had the genetic capacity to evolve earlier flowering time,

470 northern and central populations did not (Sheth and Angert 2016). This may hinder their abilities

471 to avoid hotter environments via shifts in phenology. Although phenological responses to

472 selection in a greenhouse may differ from responses to selection in the field, these previous

473 findings suggest that evolution of TPCs may be necessary for northern and central populations to

474 tolerate hotter, more thermally variable environments. Although we did not document the

475 evolution of TPCs in these populations in this study, we might detect greater evolutionary

476 responses in a resurrection experiment that uses a future set of descendent seed families.

477 However, the greater magnitude of increase in mean temperatures and temperature seasonality,

478 coupled with lower capacity for thermal adaptation puts northern and central populations of $M$.

479 cardinalis at increased risk of further population decline under continued climate change.

\section{Caveats}

481 A major caveat of this study is that we performed all experiments on plants at the seedling stage.

482 Thus, although we did not detect predicted evolutionary shifts at this early life stage, such

483 responses may still exist at later life stages in this species, for RGR or other traits such as

484 reproductive output. We recognize that we have not quantified the full fitness curves for the

485 ancestors and descendants of these populations, but many important TPC studies of a variety of 
organisms have relied on performance metrics that are partial components of total fitness (e.g.,

487 heart rate in crabs: Gaitán_

488 growth rate in nymphalid caterpillars, sockeye salmon, and tropical tree seedlings: Brett $\underline{\text { et }}$ al.

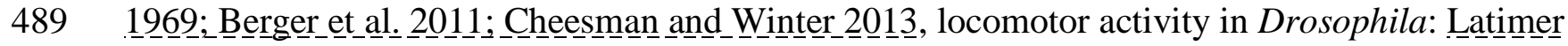

490 et al. 2011, swimming speed in tadpoles: Bartheld_et_al. 2017 , sprinting speed in skinks and

491 lizards: C_rowley__1985___Phillips et_al__2014). Importantly, our study represents an important first

492 step in quantifying physiological tolerances for ancestors and descendants of plants in a

493 resurrection study, which is complementary to many other studies of $M$. cardinalis that have

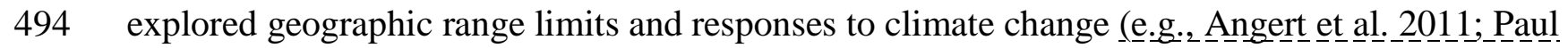

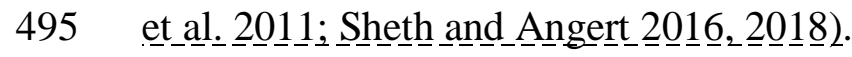

496 Our study has several additional caveats which might limit our inferences about the

497 evolution of TPCs in response to recent climate change. First, we maintained a constant

498 day/night temperature regime in growth chambers, yet it is increasingly recognized that

499 temperature variability and frequency of temperature extremes have important consequences for

500 physiological, ecological, and evolutionary processes. Second, the cohorts in our resurrection

501 study are derived from two sampling periods that are only seven years apart, which may not be

502 enough time to detect evolutionary responses in a perennial plant. Based on previously collected

503 demography data (Sheth and Angert 2018) and personal observation, we speculate that plants

504 could have plausibly completed one to seven generations between our sampling periods.

505 Strikingly, despite this potential drawback, we still detected rapid evolution of breadth in the S1

506 population, which is particularly interesting given that individuals in southern populations tend

507 to be shorter-lived than those in northern and central populations (Sheth and Angert 2018).

508 Third, if seeds that remained dormant in the seed bank for multiple years contributed to the 
509 descendant cohort in our study, they could slow the rate of evolutionary change (Hairston and De

510 Stasio 1988), but the likelihood of this is low (Sheth and Angert 2018). Fourth, the hierarchical

511 model assumes that seed families are statistically independent, however many seed families

512 shared a parent and were thus not genetically independent (Table S2). As a result, our estimates

513 of TPCs could be artificially more precise for those population/cohort combinations that had

514 fewer genetically unique families (N1 2010, N2 2010, C2 2010, and C2 2017). However, we did

515 not identify highly plausible evolutionary patterns in these populations, suggesting that greater

516 numbers of genetically non-independent families do not inflate estimates of evolutionary

517 response. Thus, future studies that consider additional life stages, performance metrics,

518 temperature regimes, and sampling years are still needed to gain a comprehensive view of long-

519 term evolutionary responses of TPCs in M. cardinalis.

521 A key question in ecology and evolutionary biology is whether populations can evolve rapidly

522 enough to keep up with the pace of climate change. Although rates of projected climate change

523 exceeded past rates of climatic niche evolution in a macroevolutionary study of vertebrates

524 (Quintero and Wiens 2013), we showed that in only seven years, breadth has decreased by over 1

$525{ }^{\circ} \mathrm{C}$ in a southern population of a perennial plant. This pattern of evolution may be due to a period

526 of drought experienced in situ from 2010 to 2017, which would have truncated the growing

527 season and reduced the range of temperatures encountered, or a trade-off in which evolution of

528 earlier flowering comes with the cost of thermal specialization. Breadth did not significantly shift

529 in any of the other five populations, and thermal optima did not significantly shift in any of the

530 six populations across the species' range, likely due to a combination insufficient time for

531 evolutionary change, weak selection, or lack of genetic variation in thermal performance. We 
532 conclude that populations, even those that are in the same region of a geographic range (e.g., the

533 two southern-edge populations in our study), can vary in their evolutionary responses to climate

534 change, having important, but often overlooked, impacts on forecasts of range shifts. More

535 importantly, our findings demonstrate that thermal performance evolution may not occur rapidly

536 in a majority of populations, even those where it is most expected. Overall, determining the

537 potential for population-level TPCs to evolve in response to recent climate change represents an

538 important step forward in understanding and predicting whether evolution can rescue populations

539 in the face of climate change.

542 We are grateful to P. Beattie, J. Smith, and A. Rosvall for help with field collections in 2010, and

543 M. Riggins, R.L. Olliff Yang, and J.E. Shay for help with field collections in 2017. We also

544 thank A.L. Angert for providing logistical support in the field. The National Park Service,

545 Bureau of Land Management, and California State Parks provided collection permits. We thank

546 J. Torres, M. Wiegmann, N. Gold, C. Yurish, E. Vtipil, and E. Coughlin for assistance with

547 pollinations, data collection, and plant care, and the North Carolina State University Phytotron \&

548 Fox Greenhouse staff for help with plant care. We thank N.C. Emery, J.W. Benning, D.N.

549 Anstett, C.D. Muir, and two anonymous reviewers for feedback on earlier versions of this

550 manuscript. This project was funded by the USDA National Institute of Food and Agriculture

551 Hatch (Project ID: 1016272) and NSF DBI (Project ID: 1523866). 
554 SNS conceived of the study; RCW and SNS designed the experiment. RCW conducted the 555 experiments and collected the data. RCW analyzed the data with the assistance of SBT. RCW 556 and SNS wrote the first draft of the manuscript and all authors contributed to editing the final 557 manuscript.

\section{References}

560 Angert, A. L. 2006. Demography of central and marginal populations of monkeyflowers (Mimulus cardinalis and M. lewisii). Ecology 87:2014-2025.

562 Angert, A. L., M. Bayly, S. N. Sheth, and J. R. Paul. 2018. Testing range-limit hypotheses using range-wide habitat suitability and occupancy for the scarlet monkeyflower (Erythranthe cardinalis). Am. Nat. 191:E76-E89.

568 Angert, A. L., S. N. Sheth, and J. R. Paul. 2011. Incorporating population-level variation in

Angert, A. L., and D. W. Schemske. 2005. The evolution of species' distributions: reciprocal transplants across the elevation ranges of Mimulus cardinalis and M. lewisii. Evolution

571 Angilletta, M. J. 2009. Thermal adaptation: a theoretical and empirical synthesis. Oxford University Press.

573 Araújo, M. B., F. Ferri $\square$ Yáñez, F. Bozinovic, P. A. Marquet, F. Valladares, and S. L. Chown. 2013. Heat freezes niche evolution. Ecol. Lett. 16:1206-1219. 
575 Bartheld, J. L., P. Artacho, and L. Bacigalupe. 2017. Thermal performance curves under daily thermal fluctuation: A study in helmeted water toad tadpoles. J. Thermal Biol. 70:80-85.

577 Bayly, M. J., and A. L. Angert. 2019. Niche models do not predict experimental demography but both suggest dispersal limitation across the northern range limit of the scarlet monkeyflower (Erythranthe cardinalis). J. Biogeogr. 46:1316-1328.

583 Brett, J. R., J. E. Shelbourn, and C. T. Shoop. 1969. Growth rate and body composition of fingerling sockeye salmon, Oncorhynchus nerka, in relation to temperature and ration size. J. Fish. Res. Bd. Can. 26:2363-2394.

Carlson, S. M., C. J. Cunningham, and P. A. H. Westley. 2014. Evolutionary rescue in a changing world. Trends Ecol. Evol. 29:521-530.

Collingham, Y. C., and B. Huntley. 2000. Impacts of habitat fragmentation and patch size upon

593 Crowley, S. R. 1985. Thermal sensitivity of sprint-running in the lizard Sceloporus undulatus: support for a conservative view of thermal physiology. Oecologia 66:219-225.

595 Davis, M. B., and R. G. Shaw. 2001. Range shifts and adaptive responses to Quaternary climate change. Science 292:673-679. 
Diamond, S. E. 2017. Evolutionary potential of upper thermal tolerance: biogeographic patterns and expectations under climate change. Ann. NY Acad. Sci. 1389:5-19.

Dickman, E. E., L. K. Pennington, S. J. Franks, and J. P. Sexton. 2019. Evidence for adaptive responses to historic drought across a native plant species range. Evol. Appl. 12:1569-

Dobzhansky, Th. 1950. Evolution in the tropics. Am. Sci. 38:209-221.

603

604

605

606

607

608

609

610

611

612

613

614

615

616

617

618

619

Etterson, J. R. 2004. Evolutionary potential of Chamaecrista fasciculata in relation to climate change. Ii. genetic architecture of three populations reciprocally planted along an environmental gradient in the Great Plains. Evolution 58:1459-1471.

Franks, S. J. 2011. Plasticity and evolution in drought avoidance and escape in the annual plant Brassica rapa. New Phytol. 190:249-257.

Franks, S. J., J. C. Avise, W. E. Bradshaw, J. K. Conner, J. R. Etterson, S. J. Mazer, R. G. Shaw, and A. E. Weis. 2008. The resurrection initiative: storing ancestral genotypes to capture evolution in action. BioScience 58:870-873.

Franks, S. J., E. Hamann, and A. E. Weis. 2018. Using the resurrection approach to understand contemporary evolution in changing environments. Evol. Appl. 11:17-28.

Franks, S. J., M. R. Sekor, S. Davey, and A. E. Weis. 2019. Artificial seed aging reveals the invisible fraction: implications for evolution experiments using the resurrection approach. Evol. Ecol., doi: 10.1007/s10682-019-10007-2.

Franks, S. J., S. Sim, and A. E. Weis. 2007. Rapid evolution of flowering time by an annual plant in response to a climate fluctuation. PNAS 104:1278-1282.

Gaitán-Espitia, J. D., L. D. Bacigalupe, T. Opitz, N. A. Lagos, T. Timmermann, and M. A. Lardies. 2014. Geographic variation in thermal physiological performance of the 

4386.

622 Geerts, A. N., J. Vanoverbeke, B. Vanschoenwinkel, W. Van Doorslaer, H. Feuchtmayr, D. Atkinson, B. Moss, T. A. Davidson, C. D. Sayer, and L. De Meester. 2015. Rapid evolution of thermal tolerance in the water flea Daphnia. Nat. Clim. Change 5:665-668.

625 Gelman, A., J. B. Carlin, H. S. Stern, D. B. Dunson, A. Vehtari, and D. B. Rubin. 2014. Bayesian data analysis. Third Edition. Chapman and Hall, London, UK.

627 Hairston, N. G., and B. T. De Stasio. 1988. Rate of evolution slowed by a dormant propagule pool. Nature 336:239-242. Hellmann. 2016. Addressing potential local adaptation in species distribution models:

Hamann, E., A. E. Weis, and S. J. Franks. 2018. Two decades of evolutionary changes in Brassica rapa in response to fluctuations in precipitation and severe drought. Evolution

635 Hampe, A., and R. J. Petit. 2005. Conserving biodiversity under climate change: the rear edge matters. Ecol. Lett. 8:461-467.

637 Higgins, J. K., H. J. MacLean, L. B. Buckley, and J. G. Kingsolver. 2014. Geographic differences and microevolutionary changes in thermal sensitivity of butterfly larvae in response to climate. Funct. Ecol. 28:982-989.

640 Hoffmann, A. A., and C. M. Sgrò. 2011. Climate change and evolutionary adaptation. Nature 
642 Hu, X.-S., and F. He. 2006. Seed and pollen flow in expanding a species’ range. J. Theor. Biol. 240:662-672.

644 Huey, R. B., and R. D. Stevenson. 1979. Integrating thermal physiology and ecology of ectotherms: a discussion of approaches. Integr. Comp. Biol. 19:357-366.

IPCC. 2013. Climate Change 2013: The Physical Science Basis. Contribution of Working Group I to the Fifth Assessment Report of the Intergovernmental Panel on Climate Change. (eds Stocker, T.F., D. Qin, G.-K. Plattner, M. Tignor, S.K. Allen, J. Boschung, A. Nauels, Y.

Janzen, D. H. 1967. Why mountain passes are higher in the tropics. Am. Nat. 101:233-249.

652 Kingsolver, J. G., S. E. Diamond, and L. B. Buckley. 2013. Heat stress and the fitness consequences of climate change for terrestrial ectotherms. Funct. Ecol. 27:1415-1423.

654 Latimer, C. a. L., R. S. Wilson, and S. F. Chenoweth. 2011. Quantitative genetic variation for thermal performance curves within and among natural populations of Drosophila serrata.

657 Lowry, D. B., J. M. Sobel, A. L. Angert, T.-L. Ashman, R. L. Baker, B. K. Blackman, Y. Brandvain, K. J. R. P. Byers, A. M. Cooley, J. M. Coughlan, M. R. Dudash, C. B.

661 Lynch, M. G., and W. Gabriel. 1987. Environmental tolerance. Am. Nat. 129:283-303.

662 Muir, C. D., and A. L. Angert. 2017. Grow with the flow: a latitudinal cline in physiology is associated with more variable precipitation in Erythranthe cardinalis. J. Evol. Biol. 30. 
664 Paul, J. R., S. N. Sheth, and A. L. Angert. 2011. Quantifying the impact of gene flow on phenotype-environment mismatch: a demonstration with the scarlet monkeyflower Mimulus cardinalis. Am. Nat. 178:S62-S79.

667 Peterson, M. L., D. F. Doak, and W. F. Morris. 2019. Incorporating local adaptation into forecasts of species' distribution and abundance under climate change. Global Change BIol. 25:775-793.

670 Phillips, B. L., J. Llewelyn, A. Hatcher, S. Macdonald, and C. Moritz. 2014. Do evolutionary constraints on thermal performance manifest at different organizational scales? J. Evol.

673 Quintero, I., and J. J. Wiens. 2013. Rates of projected climate change dramatically exceed past rates of climatic niche evolution among vertebrate species. Ecol. Lett. 16:1095-1103.

R Core Team. 2019. R: a language and environment for statistical computing. R Foundation for Statistical Computing, Vienna.

677 Rees, M., C. P. Osborne, F. I. Woodward, S. P. Hulme, L. A. Turnbull, and S. H. Taylor. 2010. Partitioning the components of relative growth rate: how important is plant size

Sheth, S. N., and A. L. Angert. 2016. Artificial selection reveals high genetic variation in phenology at the trailing edge of a species range. Am. Nat. 187:182-193.

682 Sheth, S. N., and A. L. Angert. 2018. Demographic compensation does not rescue populations at a trailing range edge. PNAS 115:2413-2418.

684 Sheth, S. N., and A. L. Angert. 2014. The evolution of environmental tolerance and range size: a comparison of geographically restricted and widespread Mimulus. Evolution 68:29172931. 
687 Socolar, J. B., P. N. Ebanchin, S. R. Beissinger, and M. W. Tingley. 2017. Shifts in time and $688 \quad$ space interact as climate warms. PNAS 114:12976-12981.

689 Stevens, G. C. 1989. The latitudinal gradient in geographical range: how so many species coexist $690 \quad$ in the tropics. Am. Nat. 133:240-256.

691 Sunday, J. M., A. E. Bates, and N. K. Dulvy. 2010. Global analysis of thermal tolerance and 692 latitude in ectotherms. Proc. R. Soc. B. 278:1823-1830.

693 Thompson, R. M., J. Beardall, J. Beringer, M. Grace, and P. Sardina. 2013. Means and extremes: 694 building variability into community-level climate change experiments. Ecol. Lett. $695 \quad 16: 799-806$.

696 Tittes, S. B., J. F. Walker, L. Torres-Martínez, and N. C. Emery. 2019. Grow where you thrive, 697 or where only you can survive? An analysis of performance curve evolution in a clade 698 with diverse habitat affinities. Am. Nat. 193:530-544.

699 Visser, M. E. 2008. Keeping up with a warming world; assessing the rate of adaptation to climate $700 \quad$ change. Proc. R. Soc. B. 275:649-659.

701 Wang, T., A. Hamann, D. Spittlehouse, and C. Carroll. 2016. Locally downscaled and spatially 702 customizable climate data for historical and future periods for North America. PLoS

$703 \quad$ ONE 11:e0156720. 
704 Table 1. Observed evolutionary change (with 95\% credible interval) in thermal optimum and

705 breadth of six populations of Mimulus cardinalis (N, C, and S designate northern, central, and

706 southern populations shown in Fig. 1A), alongside the average recent temperature anomalies

707 (difference between historical mean and each study year from 2010 to 2017; Fig. 1B-C) for each

708 population. Observed changes represent the mean differences in thermal optima and breadth

709 between descendants (derived from seed collected in 2017) and ancestors (derived from seed

710 collected in 2010) within each population. Positive values indicate that the thermal optimum or

711 breadth was higher for descendants compared to ancestors, and negative values indicate that the

712 thermal optimum or breadth was lower for descendants compared to ancestors. Highly plausible

713 differences (where credible intervals do not overlap zero) are bolded. All units are in ${ }^{\circ} \mathrm{C}$. Climate

714 data were generated for population localities (Table S1) using the ClimateWNA v5.51 software

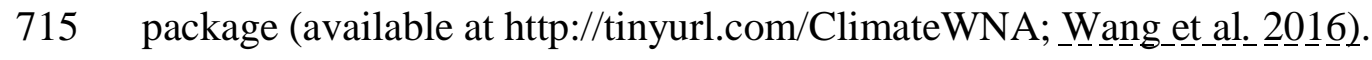

$716{ }^{\dagger}$, populations which showed significant decline from 2010 to 2014 according the a demography study by $\underline{\text { Sheth}} \underline{\text { and }} \underline{\text { and }}$

717 Angert (2019). Populations not marked with an asterisk were not included in this demography study, thus we do not

718 know their status. ${ }^{\ddagger}$, populations whose ancestors showed ample genetic variation to evolve earlier flowering times

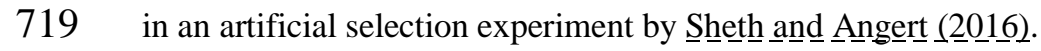




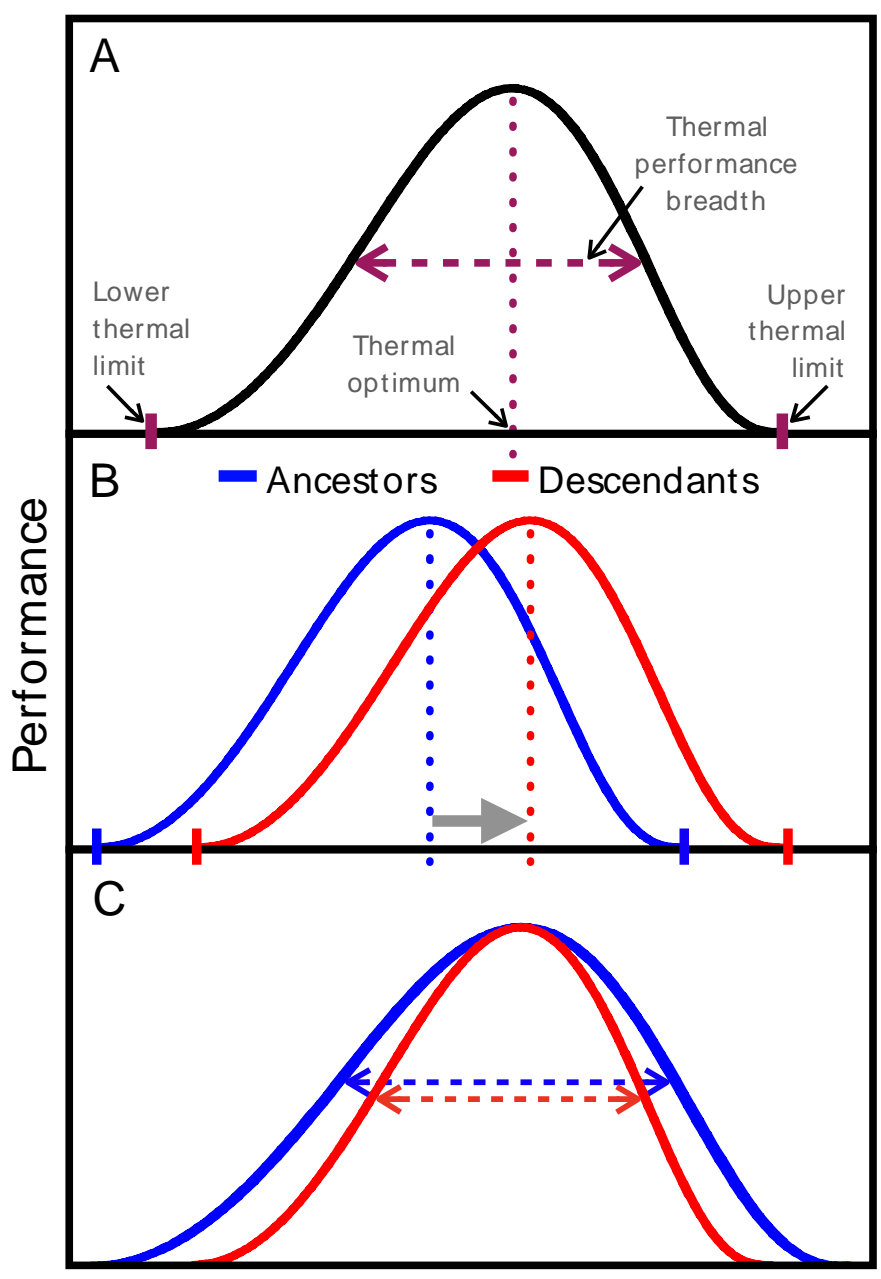

721 Figure 1. Predictions for evolution of thermal performance curves of Mimulus cardinalis in

722 response to recent climate change. A) Thermal performance curves are described by parameters

723 including the thermal optimum, thermal performance breadth, and lower and upper thermal

724 limits. B) Thermal optima of descendants should evolve to be higher than ancestors, especially in

725 northern and central populations where recent increases in maximum July temperatures have

726 been most extreme, and C) breadths of descendants should evolve to be narrower than ancestors,

727 especially in southern populations where recent decreases in temperature seasonality have been 
728 most extreme (Table 1). Images of a single S2 2010 seed family grown across the experimental

729 temperature gradient are shown below panel C, with a scale for size. 

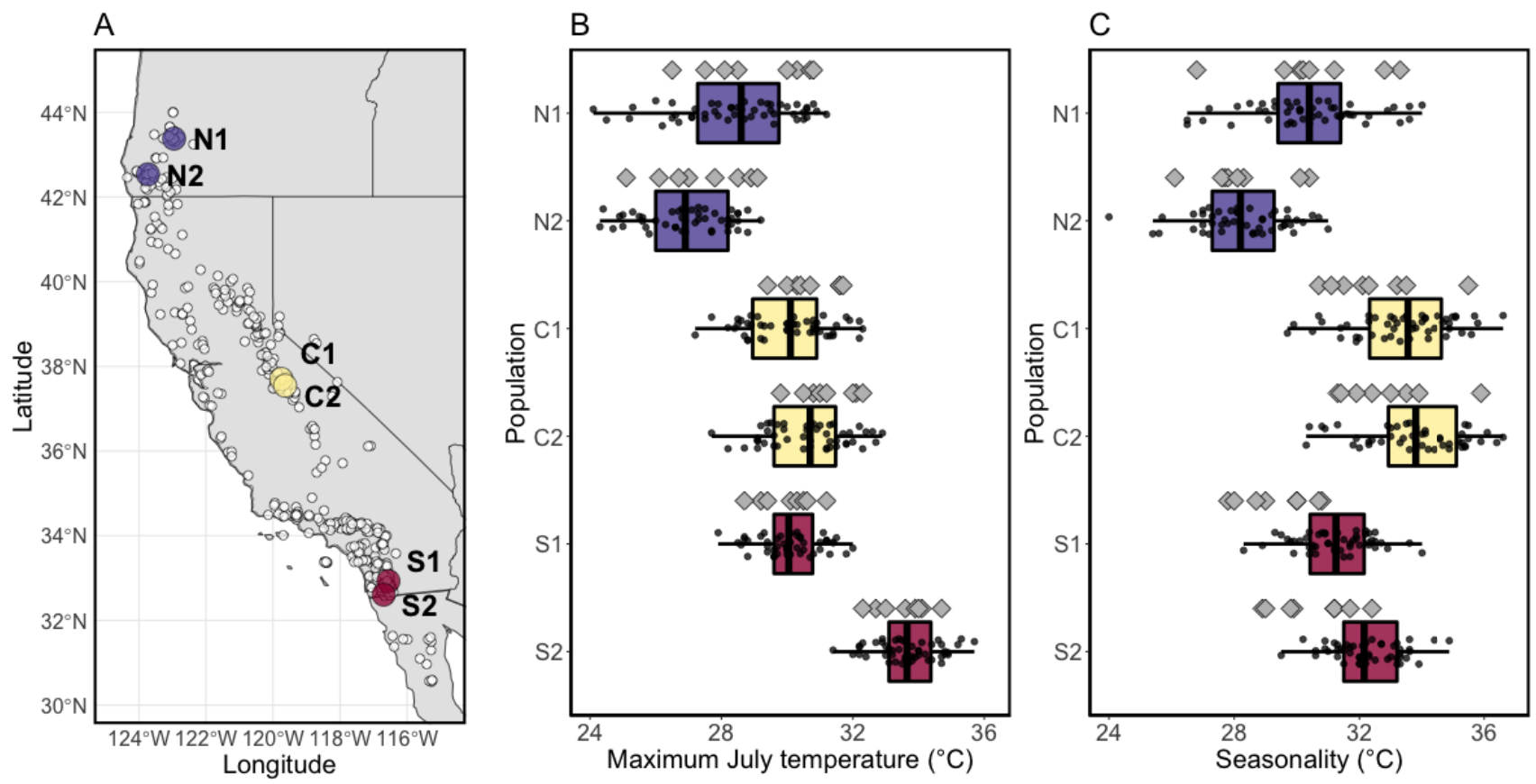

731 Figure 2. A) Map of seed collection sites of two northern-edge (purple), two central (yellow),

732 and two southern-edge (red) populations of Mimulus cardinalis, superimposed on herbarium

733 specimen locations (white circles; A__ngert_et__al__2018). Recent (2010-2017) B) maximum July

734 temperatures and C) temperature seasonality for each population (gray diamonds), superimposed

735 on boxplots of historical values (1951-2000). Climate data are described in Table S1. 


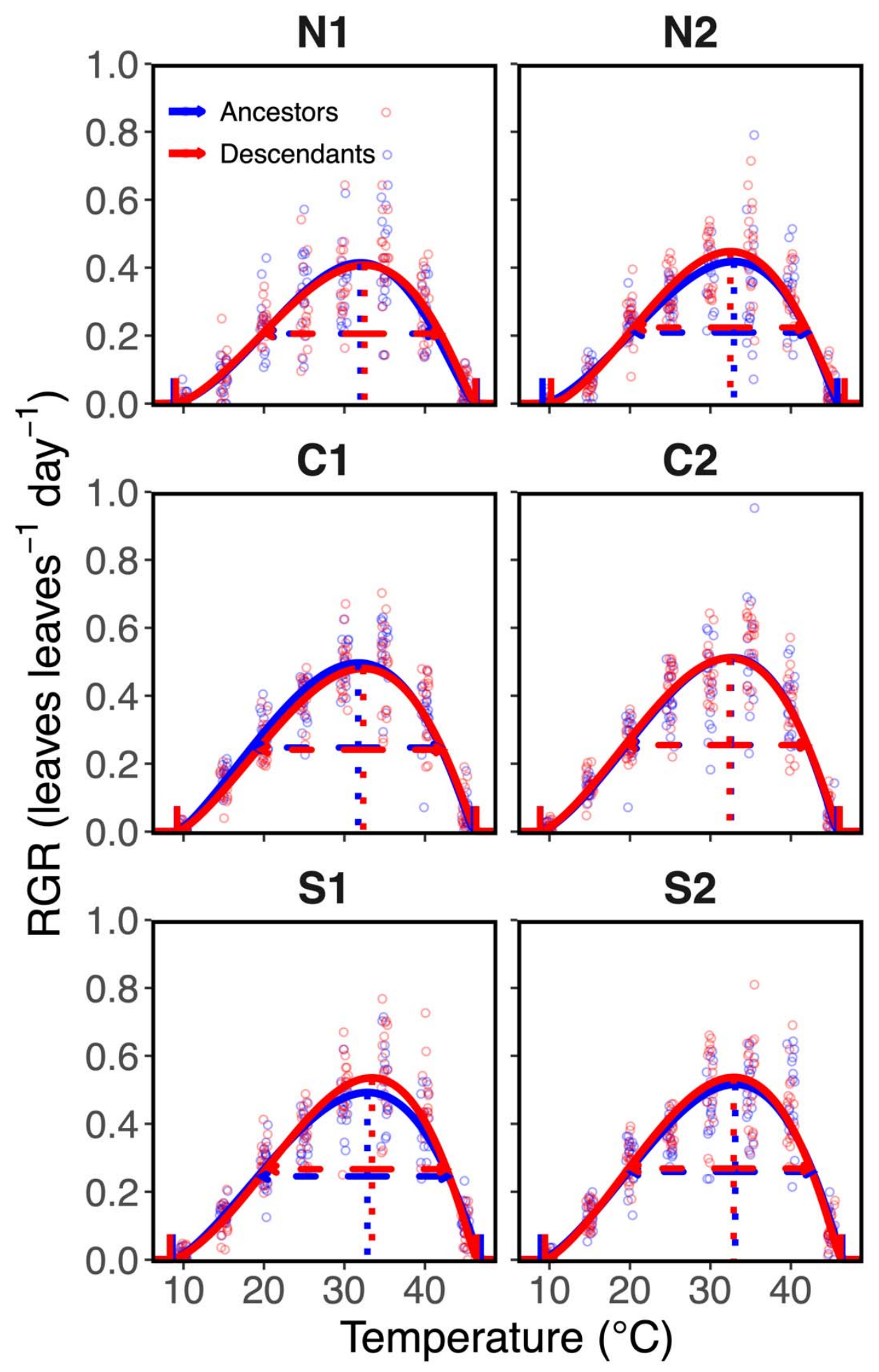

737 Figure 3. Thermal performance curves of the ancestors and descendants in each of six Mimulus

738 cardinalis populations. Vertical dotted lines represent thermal optima, horizontal dashed lines 
739 represent thermal performance breadths (range of temperatures across which plants achieve 50\%

740 of maximum growth), and notches on the x-axis indicate lower and upper thermal limits. The x-

741 axis represents daytime temperatures in growth chambers. Open points represent family means at

742 each temperature, which are horizontally scattered around each temperature at random to aid

743 visualization. 

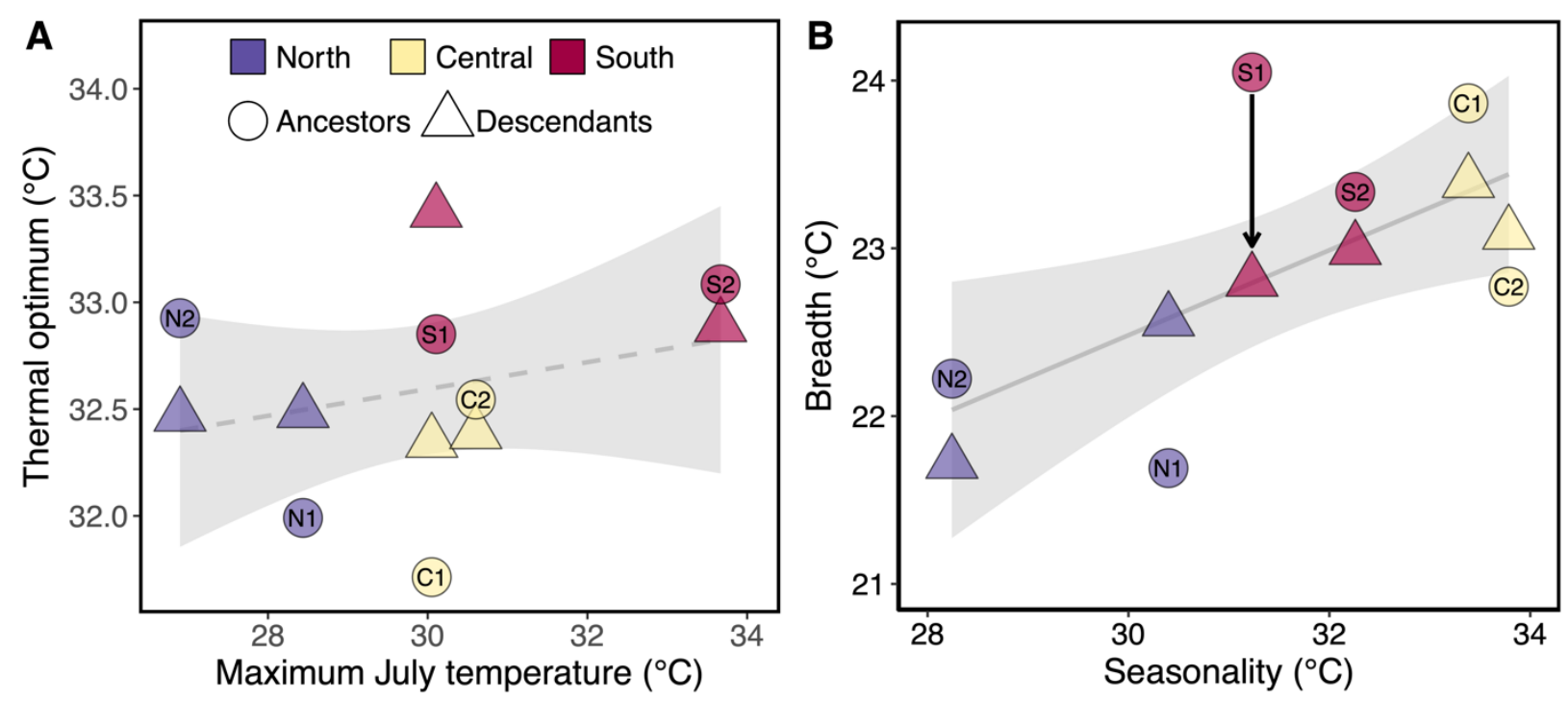

745 Figure 4. Variation in A) thermal optima across historical mean temperatures and B) breadth

746 across historical seasonality. Thermal optima and breadth values represent mean estimates

747 derived from a hierarchical Bayesian model, and are colored by the region where populations

748 occur within the species' geographic range (Fig. 2A). The arrow indicates highly plausible

749 evolutionary change from 2010 (ancestors) to 2017 (descendants) (Table 1). Population codes

750 are overlaid onto ancestral values. The regression in panel A was not significant at $\alpha=0.05$

751 (dashed line), but breadth significantly increased with seasonality (solid line; see results in the

752 main text). Shaded intervals represent $95 \%$ confidence intervals. Climate data are described in 753 Table S1. 